\title{
Nanoindentation of Bridgman YBCO samples
}

\author{
J.J. Roa ${ }^{\text {a,b,*, K. Konstantopoulou }}{ }^{\text {c }}$, E. Jiménez-Piqué ${ }^{\text {d }}$, V. Martín ${ }^{\text {d, M. Segarra }}{ }^{\text {, J.Y. Pastor }}{ }^{\text {c }}$ \\ 'Institut P' (UPR 3346), Département de Physique et Mécanique des Matériaux, CNRS, Université de Poitiers, ENSMA, Boulevard Marie et Pierre Curie, \\ SP2MI, Téléport 2, BP 30179, F-86962 Futuroscope Chasseneuil Cedex, France \\ ${ }^{\mathrm{b}}$ Departament de Ciencia dels Materials i Eng. Metal.lurgica, Universitat de Barcelona, C/Martí i Franquès, I, 08028 Barcelona, Spain \\ 'Departamento de Ciencia de Materiales, E.T.S. de Ingenieros de Caminos, Canales y Puertos, CISDEM (UPM-CSIC), \\ Universidad Politécnica de Madrid, C/Profesor Aranguren s/n, 28040 Madrid, Spain \\ ${ }^{\mathrm{d}}$ Departamento de Ciencia de los Materiales e Ing. Metalúrgica, Universidad Politécnica de Cataluña 647 (ETSEIB), 08028 Barcelona, Spain
}

\begin{abstract}
In this study, the mechanical properties of $\mathrm{YBa}_{2} \mathrm{Cu}_{3} \mathrm{O}_{7-x}$, obtained by the Bridgman technique, were examined using a Berkovich tip indenter on the basal plane $\left(\begin{array}{lll}0 & 0 & 1\end{array}\right)$. Intrinsic hardness was measured by nanoindentation tests and corrected using the Nix and Gao model for this material. Furthermore, Vickers hardness tests were performed, in order to determine the possible size effect on these measurements. The results showed an underestimation of the hardness value when the tests were performed with large loads. Moreover, the elastic modulus of the Bridgman samples was $128 \pm 5 \mathrm{GPa}$. Different residual imprints were visualised by atomic force microscopy and a focused ion beam, in order to observe superficial and internal fracturing. Mechanical properties presented a considerable reduction at the interface. This effect could be attributed to internal stress generated during the texturing process. In order to corroborate this hypothesis, an observation using transmission electron microscopy was performed.
\end{abstract}

Keywords: Superconductor material; YBaCuO; Bridgman; Nanoindentation

\section{Introduction}

$\mathrm{YBa}_{2} \mathrm{Cu}_{3} \mathrm{O}_{7-\delta}(\mathrm{YBaCuO})$ is a superconducting material with a perovskite structure which played a pivotal role in arriving at our current understanding of superconductivity and its scientific development. The material is widely employed for various applications, including high temperature superconductor (HTSC) SQUIDs at liquid nitrogen operation temperatures [1], non-destructive evaluation of defects in complex computer chips [2] and aircraft [3], among others. In order to produce devices for these applications, different stress conditions are applied to the material. Furthermore, when the devices are operating, they are required to support certain working conditions. For this reason, accurate determination of their mechanical properties is important in order to develop better manufacturing processes and to assure structural integrity during service. In addition, descriptions of the deformation mechanisms present in the material and their influence on its mechanical properties are very important in order to understand its mechanical and deformation behaviour.

$\mathrm{YBaCuO}$ presents several important advantages over other ceramic superconductors (i.e. bismuth strontium calcium copper oxide): (i) it is the only stable four-element compound known at the moment with a critical temperature $\left(T_{C}\right)$ above $77 \mathrm{~K}$; (ii) it includes neither toxic elements nor volatile compounds, and production of single crystals is relatively easy; and (iii) it presents higher isotropy than other HTSC materials and it carries higher current densities at higher magnetic fields.

One of the reasons for the remarkable properties of $\mathrm{YBaCuO}$ is its oxygen-deficient orthorhombic Pmmm structure [4]. This cell unit is composed of three perovskite cells, where the Ba and $\mathrm{Y}$ cations take the central positions and copper atoms with different coordination numbers are arranged in two structural blocks with different electronic properties: copper chains and planes $[5,6]$. The $\mathrm{YBaCuO}$ lattice has a laminar structure with a high $c / a$ ratio. 
Nanoindentation is a very versatile technique for making hardness $(H)$ and elastic modulus $(E)$ measurements from a small volume of material, similar to that produced in research laboratories. One of the main advantages of nanoindentation is that it enables mechanical properties to be determined by analysing the load $(P)$-displacement $(h)$ curves without the need to visualise the residual imprint.

System response depends on the kind of indenter used and the structure and composition of the material. Using sharp indenters (generally Berkovich or Vickers), plastic deformation is produced from the beginning of the contact. Blunt indenters (generally spherical or conical) produce an elastic deformation of the material. The high levels of hydrostatic compression generated during normal indentation and sliding contacts facilitate activation of different plastic deformation mechanisms such as dislocations, twining, and kink bands [7]. Contactinduced deformation mechanisms are highly dependent on the crystallographic orientation of the surface evaluated. Moreover, the applied load employed during the experiments also affects the stress distribution under the imprint and thus the preference for activating particular slip systems. However, the literature on the mechanical properties of this material at micro/nanometric scale, particularly as regards pure $\mathrm{YBaCuO}$ textured by the TSMG technique, is scarce.

The mechanical properties (hardness, elastic modulus and stress-strain curves) of YBCO Bridgman samples have been reported in previous studies $[8,9]$. Some authors have studied the hardness, elastic modulus and yield strength of $\mathrm{YBaCuO}$ samples textured via the Bridgman technique, at room or at cryogenic temperatures, also known as the working temperature $\left(T_{\mathrm{w}}\right)$. Some mechanical properties, such as hardness, elastic modulus and yield strength have been examined by nanoindentation at room temperature, yielding values of 8.9-15.2 GPa, 173-204 GPa and $3.5 \mathrm{GPa}$, respectively $[8,9]$. Several studies performed at cryogenic temperature [10] exhibit that the mechanical properties tend to be higher due to the nitrogen go inside of the preexisting cracks yielding a homogeneous material. In this study we focused our attention with this kind of materials at room temperature because their behaviour is much brittle that at cryogenic temperature. Moreover, the study of the mechanical at room temperature it is possible to know the quality of the samples before the oxygenation process.

This wide spread is due to the fact that different techniques can be used to measure elastic modulus: the pulsed echo technique [11], Vickers indentation [12], ultrasonic techniques [13] or even force spectroscopy-AFM [14,15], but the results are not directly comparable since the measurement conditions are quite different.

The nanoindentation technique provides information about the contact mechanisms taking place in the material. In brittle materials indented with a sharp tip such as a Berkovich tip, surface examination of the residual imprints has revealed the appearance of several typical features, including deformation effects (sink-in [16,17], microcracks or damage inside the imprint [18]), and some fracture mechanisms, for example radial cracks emanating from the corners of the imprints [19] and chipping [20].
The purpose of this study was to characterise the mechanical properties (hardness and elastic modulus) of $\mathrm{YBaCuO}$ samples textured by the Bridgman technique at room-temperature, using micro- and nano-indentation techniques. The indentation size effect (ISE) was determined and excluded from the final properties given. The different brittle surface effects activated by nanoindentation of the $(001)$ of $\mathrm{YBaCuO}$ are described in detail and surface effects in the residual imprints were visualised by atomic force microscopy (AFM), and focused ion beams (FIB).

\section{Experimental procedure}

\subsection{Powder preparation and sintering process}

YBaCuO powders were prepared by the PVA method [21]. The ratio used here $(69 \%, \mathrm{w} / \mathrm{w}, \mathrm{Y}-123 ; 30 \%, \mathrm{w} / \mathrm{w}, \mathrm{Y}-211$; and $1 \%$, w/w, $\mathrm{CeO}_{2}$ ) has been demonstrated to maximise superconducting properties such as critical current density [22]. The calcined powder was deagglomerated by ball milling in agate mortar. The powder was compressed under isostatic pressure as a bar and inserted into the furnace at the peritectic temperature $\left(1010^{\circ} \mathrm{C}\right)$; the semisolid bars were hung inside the furnace and moved at a constant rate of $1 \mathrm{~mm} / \mathrm{h}$ through a region with an axial temperature gradient of $20 \mathrm{~K} \mathrm{~cm}^{-1}$ at the peritectic temperature [23]. After the texturing process, $\mathrm{YBaCuO}$ pieces were oxygenated in a horizontal furnace at $450{ }^{\circ} \mathrm{C}$ for $240 \mathrm{~h}$ [22]. From the oxygenated pieces with a common $c$-axis tilt of $45^{\circ}$ from the longitudinal direction of the bar, small pieces of $2 \mathrm{~mm}$ height were cut along the $a b$-plane or $\left(\begin{array}{lll}0 & 0 & 1\end{array}\right)$ [23]. Prior to nanoindentation, the sintered specimens were polished with diamond suspensions of $6,3,1$, and $1 / 4 \mu \mathrm{m}$ and colloidal silica dissolved in isopropanol. Thin TEM foils were prepared by slow, mechanical polishing down to $20 \mu \mathrm{m}$ prior to being argon ion-thinned using a precision ion-polishing system (PIPS) from Gatan.

\subsection{Microstructural characterisation}

The microstructure of the samples was studied using a CM30 Philips transmission electron microscopy (TEM). Different observations were performed on $\mathrm{YBaCuO}$ samples in the $a b$-plane and $c$-axis.

\subsection{Mechanical properties}

\subsubsection{Nanoindentation tests}

Nanoindentation test were performed using a Nano IndenterXP System (Agilent Technologies) with continuous stiffness measurements (CSM) at $2 \mathrm{~nm}$ harmonic displacement and $45 \mathrm{~Hz}$ frequency. Strain rate was held constant at $0.05 \mathrm{~s}^{-1}$. Experiments were performed on the basal plane ( $a b$-plane or 001 ) at room temperature using a Berkovich tip. The Berkovich tip function area was calibrated using a fused silica standard [24], assuming a elastic modulus of $72 \mathrm{GPa}$. Nanoindentation tests were performed at ten different maximum loads $(1,2,3,5,10,15,30,100,300$ and $500 \mathrm{mN})$ 
with 100 indents for each load. For small loads (in the range of $1-10 \mathrm{mN}$ ), nanoindentation were performed on a single $\mathrm{YBaCuO}$ phase, either on the Y-123 phase or on the Y-211 phase. The load-indentation depth curves were recorded for each nanoindentation test, and the hardness and elastic modulus were determined from the elastic unloading curve using the Oliver and Pharr method [8,24,25].

The residual imprints at very low applied load (lower than $10 \mathrm{mN}$ ) for each sample were observed by Atomic Force Microscopy (AFM, 'Digital Instruments Multimode') in tapping mode, and the images were processed using WSxM software [26]. The different imprints obtained at loads higher than $10 \mathrm{mN}$ were observed using a Field Emission Scanning Electron Microscopy (FE-SEM) in order to observe the different fracture mechanisms activated as a result of the field stress generated during the indentation process. The damage produced under the residual Berkovich imprint of $\mathrm{YBaCuO}$ samples textured by Bridgman technique was characterised using a dual beam FIB/SEM (FIB Strata DB235). The residual imprint was cross-sectioned along the $c$-axis ( 110$)$ to evaluate the damage after indentation. A thin platinum layer was deposited on the sample prior to FIB machining in order to minimise ion-beam damage. $\mathrm{A} \mathrm{Ga}^{+}$ion source was used to mill the surface at a voltage of $30 \mathrm{kV}$. The final polishing of the cross-sections was performed at $10 \mathrm{pA}$.

\subsubsection{Microindentation tests}

Microindentation tests were performed on the polished $a b$ plane $\left(\begin{array}{lll}0 & 0 & 1\end{array}\right)$ using an ISOSCAN OD Galileo Durometer. This study was performed using a Vickers diamond tip indenter. The maximum applied load was 3 and 4 N. Fifty indentations were made on each sample, and the results were averaged.

\section{Results and discussion}

\subsection{Microstructure of $\mathrm{YBaCuO}$ samples}

$\mathrm{YBaCuO}$ samples present different kinds of microstructural defects inside the single crystal, such as twin boundaries and dislocations. Each of these defects will be explained in more detail below:

- Twin boundaries $\rightarrow$ this kind of defect is generated during the tetragonal to orthorhombic phase transition during the sintering and texture process due to the high temperatures (see Fig. 1a) [27].

- Dislocations $\rightarrow$ bulk samples textured by Bridgman technique exhibit many dislocations in the $\mathrm{YBa}_{2} \mathrm{Cu}_{3} \mathrm{O}_{7-x}$ phase (or Y-123; see Fig. 1b). This phase is highly anisotropic, being confined to the $a b$-plane. Lubenets et al. [28] reported that strong covalent and ionic bonds create high Peierls barriers, which constrain dislocation mobility in $\mathrm{YBaCuO}$ single crystals. Fig. 1c depicts some dislocations placed primarily on the grain boundary between $\mathrm{Y}-123$ and $\mathrm{Y}_{2} \mathrm{BaCuO}_{5}$ (or Y-211). These dislocations could be attributed to three different factors: (i) compressive strain during the undercooling treatment in the texturing process, (ii) the different thermal expansion coefficients between the matrix and the different inclusions (from $20^{\circ} \mathrm{C}$ up to $900^{\circ} \mathrm{C}$, these are $0.00124 \mathrm{~K}^{-1}$ and $0.00170 \mathrm{~K}^{-1}$ for $\mathrm{Y}-211$ and $\mathrm{Y}-123$, respectively [29]), and
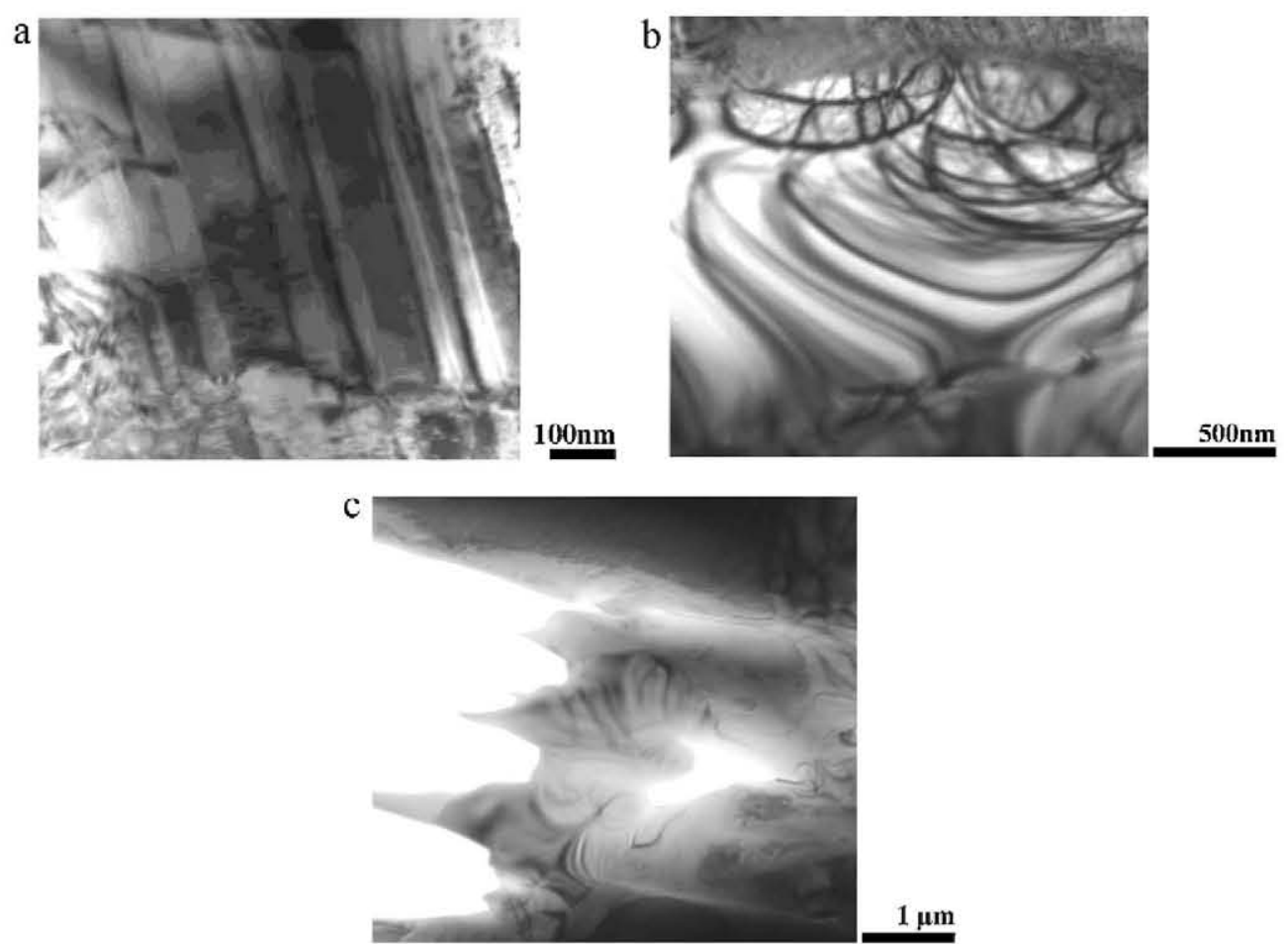

Fig. 1. TEM micrographs of different defects present in $\mathrm{YBaCuO}$ samples textured by Bridgman technique. (a) Twinning process in the $c$-axis, (b) dislocations on the maximum anisotropy plane ( $a$-plane), and (c) residual stress due to the interaction between Y-123 and Y-211. 


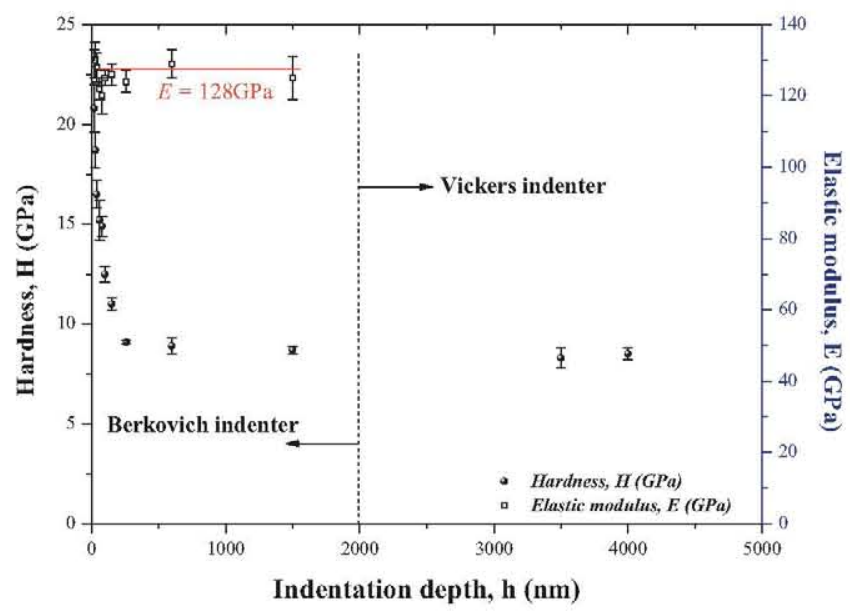

Fig. 2. Hardness and elastic modulus evolution at different applied loads for $\mathrm{YBaCuO}$.

(iii) the stress thought to result from the incorporation of Y-211 decomposition products into the matrix.

\subsection{Hardness and elastic modulus determination}

The evolution of both hardness and elastic modulus for the interphase between Y-123 and Y-211 phases in the $a b$-plane at different indentation depths for $\mathrm{YBaCuO}$ samples textured by Bridgman techniques can be observed in Fig. 2. This figure shows that the elastic modulus did not vary significantly with the indentation depth, yielding a constant value of $128 \pm 5 \mathrm{GPa}$. As can also be seen in Fig. 2, hardness values increased as indentation size decreased. This is known as the indentation size effect (ISE). Many reports of ISE are actually due to several factors which can modify this parameter, such as surface layers that were not accounted for or poor tip shape calibration [30]. Such indentation experiments can be described by the Nix and Gao equation [31]:

$\frac{H}{H_{\mathrm{o}}}=\sqrt{1+\frac{h^{*}}{h}}$

where $H$ is hardness for a given indentation depth, $H_{\mathrm{o}}$ is hardness in the absence of strain gradients, $h^{*}$ is a length scale parameter, and $h$ is depth of indentation. Eq. (1) was used to determine the intrinsic hardness of $\mathrm{YBaCuO}$ samples textured by Bridgman technique. Fig. 3 shows the variation of $H^{2}$ versus $1 / h$, where the experimental data were well fitted by the function proposed in the Nix and Gao model. The intrinsic hardness value of $\mathrm{YBaCuO}$ can be obtained directly from the fit shown in Fig. 3, yielding $H_{0}=8.8 \pm 0.3 \mathrm{GPa}$. The intrinsic hardness is in good agreement with the value obtained directly from the Oliver and Pharr method at large depths. This equation allows to extract a constant hardness value (form very low load up to microhardness range) without the contribution of the different superficial defects as roughness, cracks, and others, which produce a strongly modification of this value.

When the residual imprint was greater than the size of the different secondary phases (Y-211) (around $10 \mathrm{mN}$ of

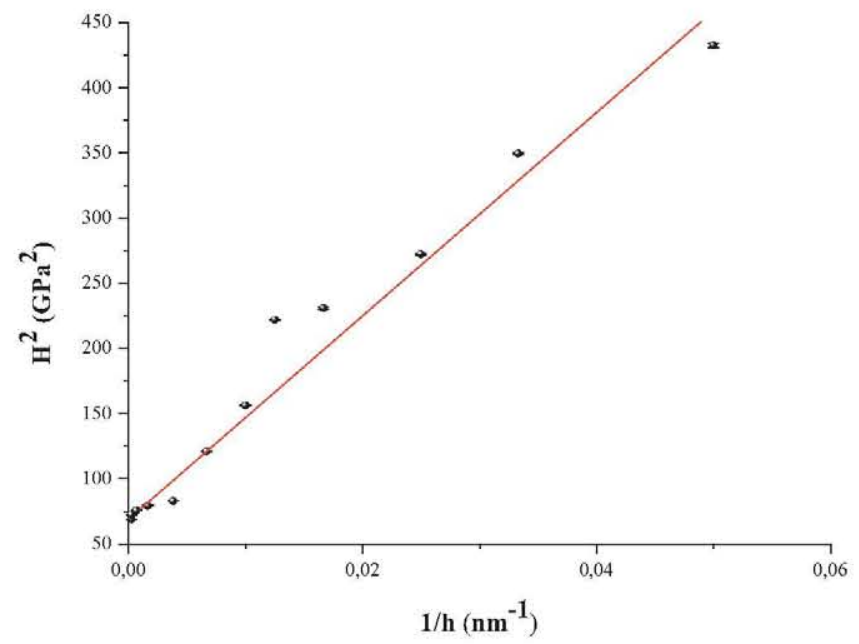

Fig. 3. Representation of $H^{2}$ versus $l / h$ for $\mathrm{YBaCuO}$, linear regression corresponds to that described in Eq. (1).

indentation depth [8] for this kind of material), hardness was similar (around $8.5 \mathrm{GPa}$ ), and also similar to the hardness of the Y-123 phase. These values are in agreement with previous studies reported by Lim and Chaudhri [32], using microindentation tests at $10 \mathrm{mN}$ of applied load, and Verdyan et al. [33], conducting hardness testing for orthorhombic $\mathrm{YBaCuO}$ thin films at applied loads of 0.1 and $0.9 \mathrm{mN}$. Furthermore, the elastic modulus value shown in Fig. 1 ( $E=128 \pm 5 \mathrm{GPa}$ ), is in good agreement with Roa et al. [8,9] and Alford et al. [34], obtained using nanoindentation and bending methods, respectively.

\subsection{Residual imprints visualisation}

Fig. 4 shows the indentation imprints obtained by applying $30 \mathrm{mN}$ on the $a b$-plane, and also depicts the Y-211 inclusions

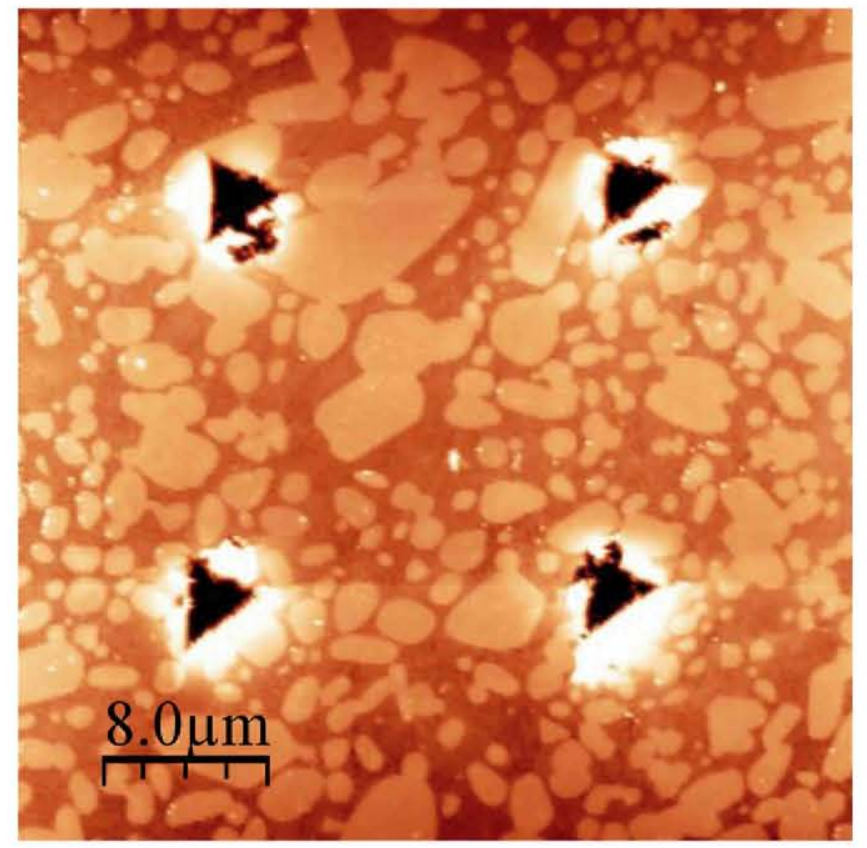

Fig. 4. AFM image (topography mode) of nanohardness imprints performed at $30 \mathrm{mN}$ of applied load. 


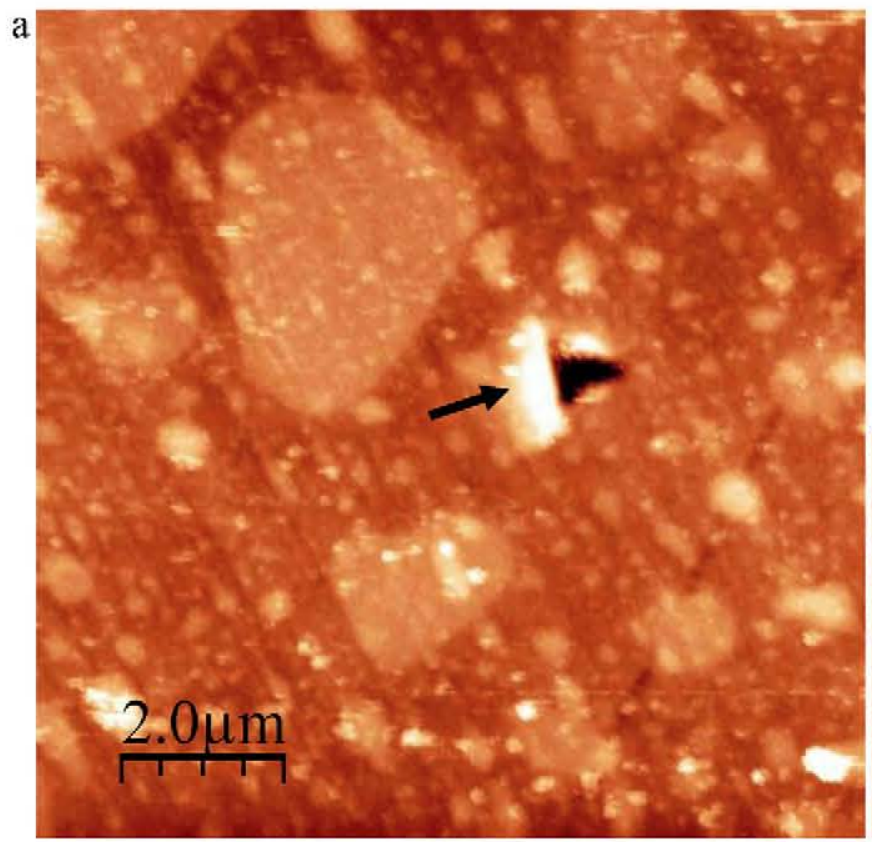

\section{Inset}

b

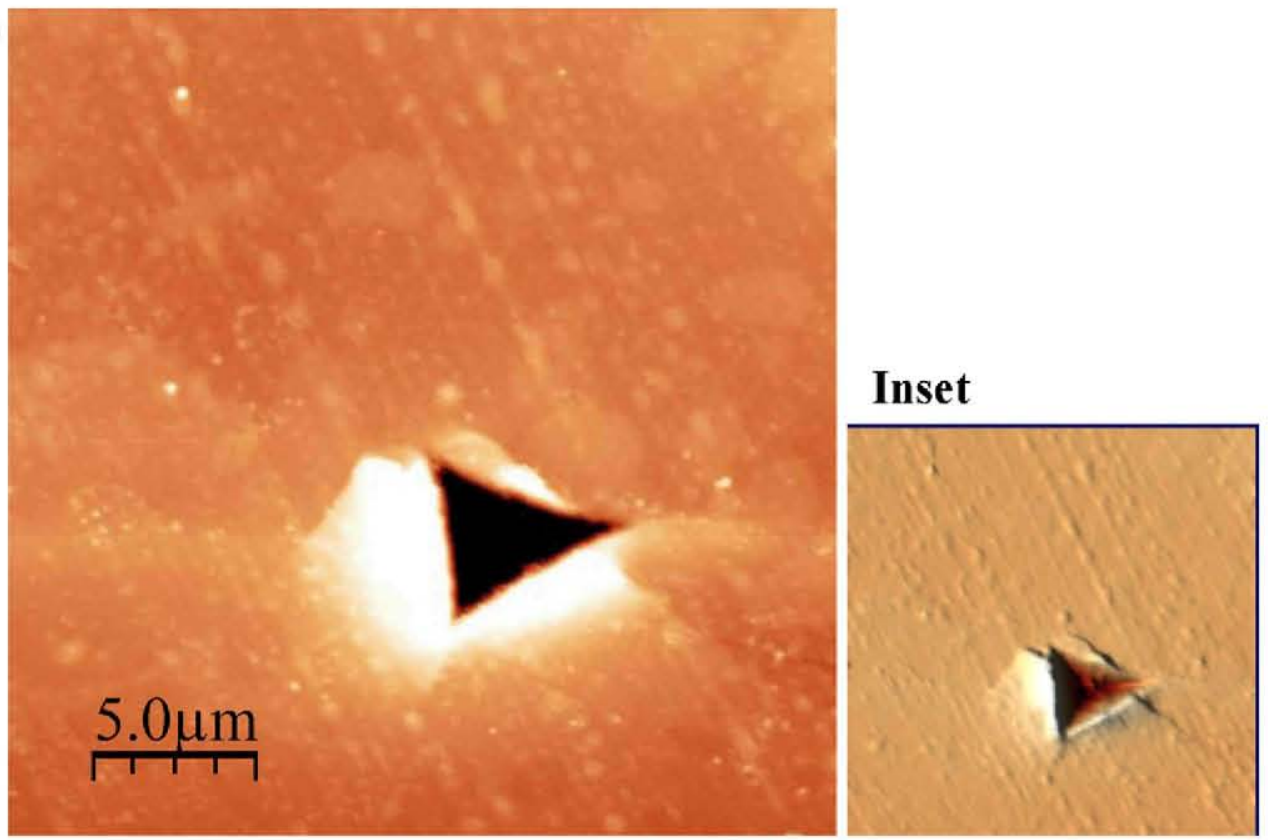

Fig. 5. AFM image (topography mode) of a residual nanoindentation imprint in the YBaCuO-Bridgman sample. Inset 1 gives the 3D image of the studied region. (a) Indentation performed at $5 \mathrm{mN}$ of applied load and (b) indentation performed at $100 \mathrm{mN}$.

which, were distributed heterogeneously throughout the textured sample; consequently, they could not be isolated the mechanical properties at higher applied loads. The different imprints in this figure have been homogeneously separated (around $10 \mu \mathrm{m}$ ) in order to avoid modification of the mechanical properties as a result of the influence of the elastic and plastic field stress generated during the indentation process.

Fig. 5a shows one of the residual imprints obtained in the $\mathrm{YBaCuO}$ composite at $5 \mathrm{mN}$. The topographic image of AFM shows a heterogeneous distribution of secondary phases with different grain sizes (from several $\mathrm{nm}$ up to $5 \mu \mathrm{m}$ ). On the lefthand side of the imprint (see black arrows), the figure also depicts chipping due to field stress relaxation after the unloading process. The inset presented in this figure shows the 3D image. The chipping effect may have been activated during indenter unloading, as a result of lateral cracks created by the high deformation fields [35]. For this applied load, the residual imprint was smaller than some of the inclusions distributed inside the matrix, and thus the mechanical properties for each phase could be measured individually. Fig. 5 b shows a residual imprint obtained at $100 \mathrm{mN}$ of applied load, and deformation in the vicinity of the residual imprint can be observed, in addition to chipping and radial cracks at the corner of the imprint. These radial cracks were attributed to the 


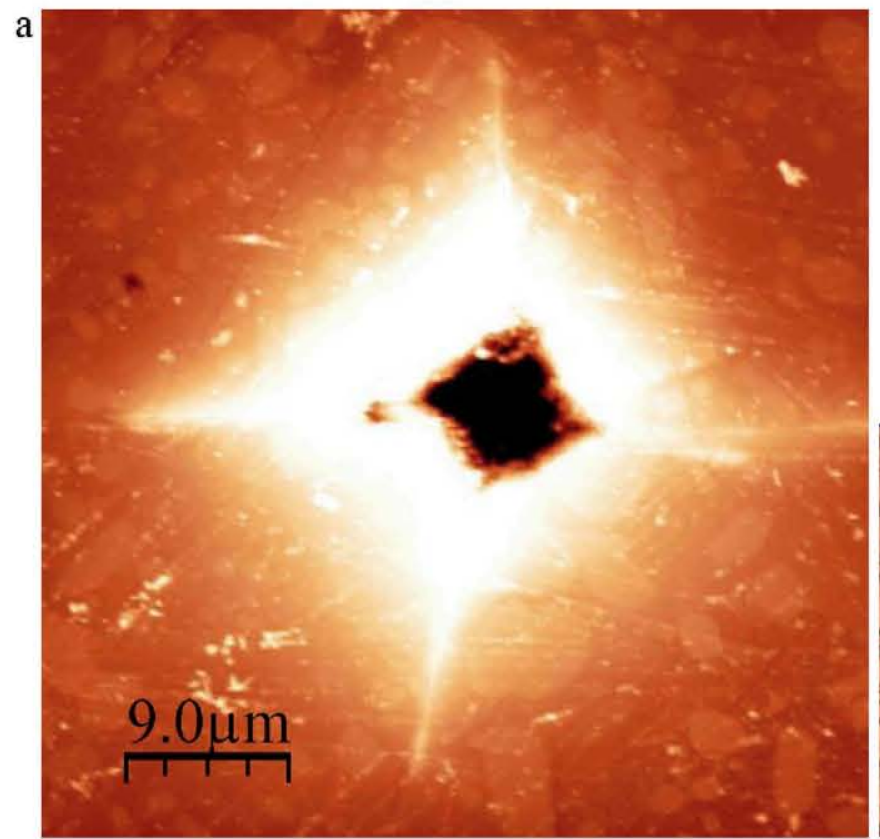

\section{Inset}
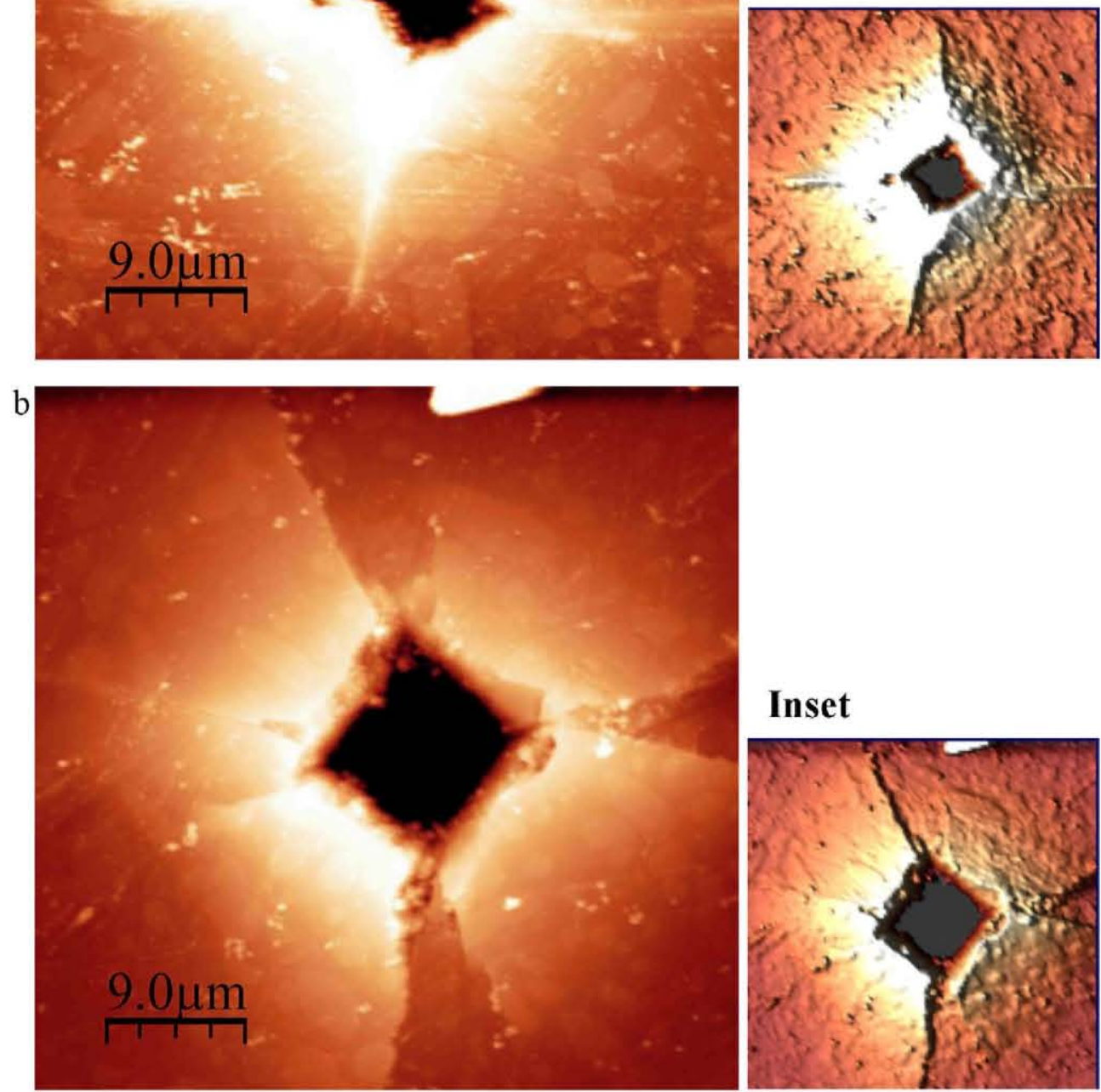

\section{Inset}

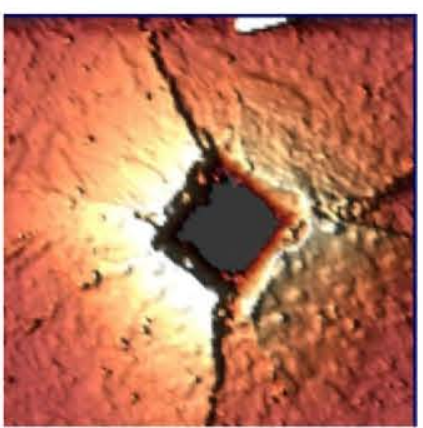

Fig. 6. AFM image (topography mode) of a residual microindentation imprint in the $\mathrm{YBaCuO}$-Bridgman sample. Inset 1 gives the 3D image of the studied region. (a) Indentation performed at $3.5 \mu \mathrm{m}$ of indentation depth (around $3 \mathrm{~N}$ of applied load) and (b) indentation performed at $4 \mu \mathrm{m}$ of indentation depth (around $4 \mathrm{~N}$ of applied load).

different levels of residual elastic-plastic contact stresses between the tip and the sample [36]. When the applied load was higher than $10 \mathrm{mN}[8]$, the size of the residual imprint was greater than the size of the different inclusions, and the mechanical properties for each phase could not be isolated; it was only possible to obtain the response of the composite. These results are in good agreement with a previous study published by Roa et al. [8].

Fig. 6 shows the AFM (topography and 3D mode) images of some residual imprints obtained at 3 and $4 \mathrm{~N}$ of applied load using the Vickers indentation technique. Both figures show a brittle behaviour, with longitudinal cracks appearing in each corner of the imprints. Moreover, a pile-up effect can be observed surrounding the residual imprint. Due to the asymmetrical micro-cracking surrounding the Vickers imprint, it was not possible to estimate the fracture toughness, $K_{\text {IC. }}$.

\subsection{Fracture mechanisms under the imprint}

One of the imprints obtained at $10 \mathrm{mN}$ of applied load was visualised by FIB, in order to better understand the fracture 

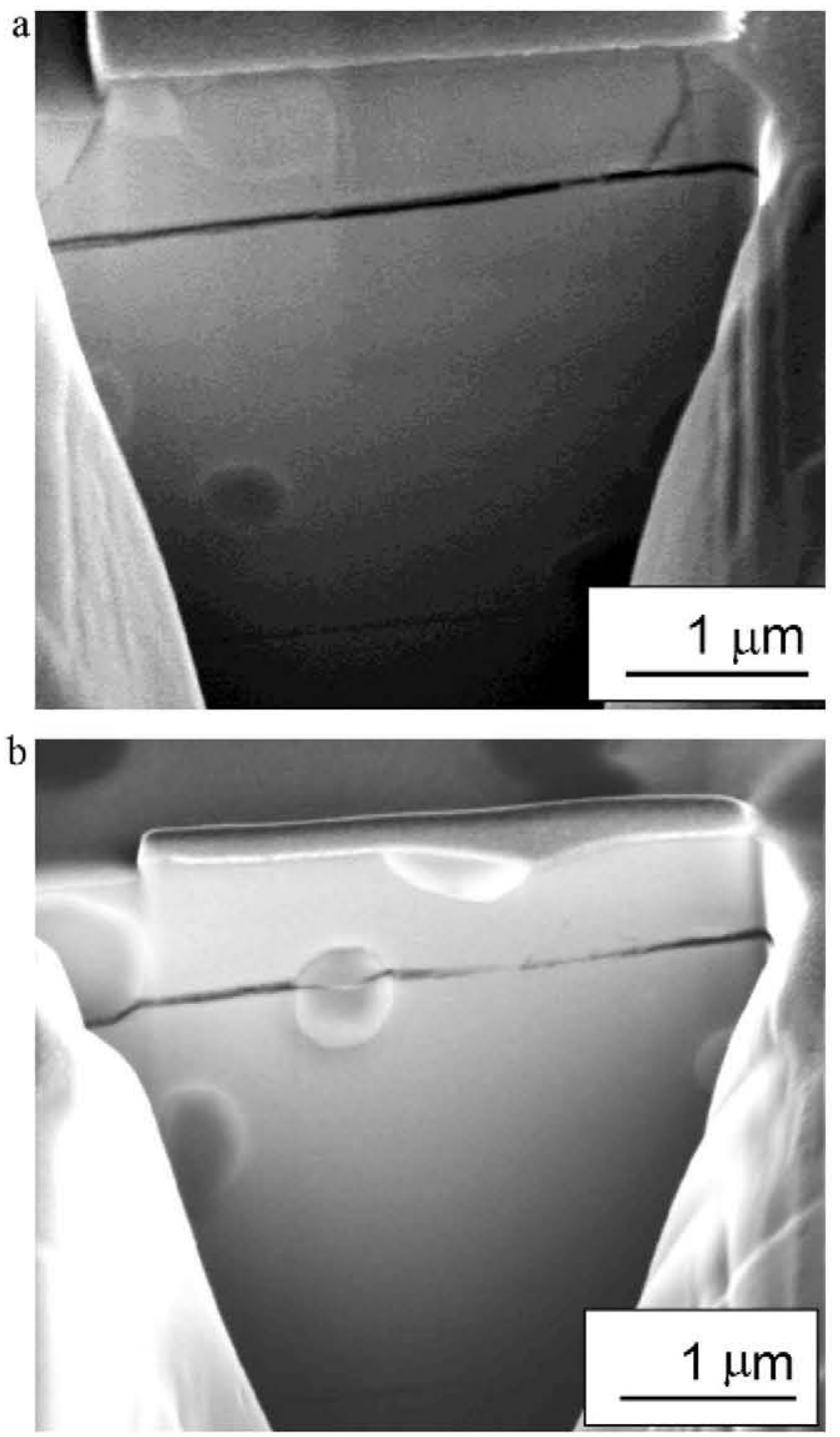

Fig. 7. Cross-sectioning and imaging of damage under an indentation using FIB/SEM for a Bridgman sample performed at $100 \mathrm{~nm}$ of indentation depth. (a) Trench milled at high ion beam current in front of the residual indentation imprint and (b) cross-sectioning in the middle of the imprint.

mechanisms that take place under this field stress (see Fig. 7). Different cracks, which originated during the texture and oxygenation process, appeared below the indentation imprint because they were almost parallel to the $a b$-plane [37]. Each image showed a heterogeneous distribution of Y-211 inclusions with different sizes, from 1 to $5 \mu \mathrm{m}$. Lastly, no cracks or failure events were visible under the nanoindentation imprint. As can be seen in the TEM images (Fig. 8), the stress field generated during the undercooling process produced a high number of dislocations in the interphase between Y-123 and Y-211, indicating that high local internal stresses were produced when neighbouring parts of the crystal were forced to change their specific volume. This can occur because neighbouring regions expand or contract as a result of differences in thermal gradients, changes in composition or changes in lattice structure [38].
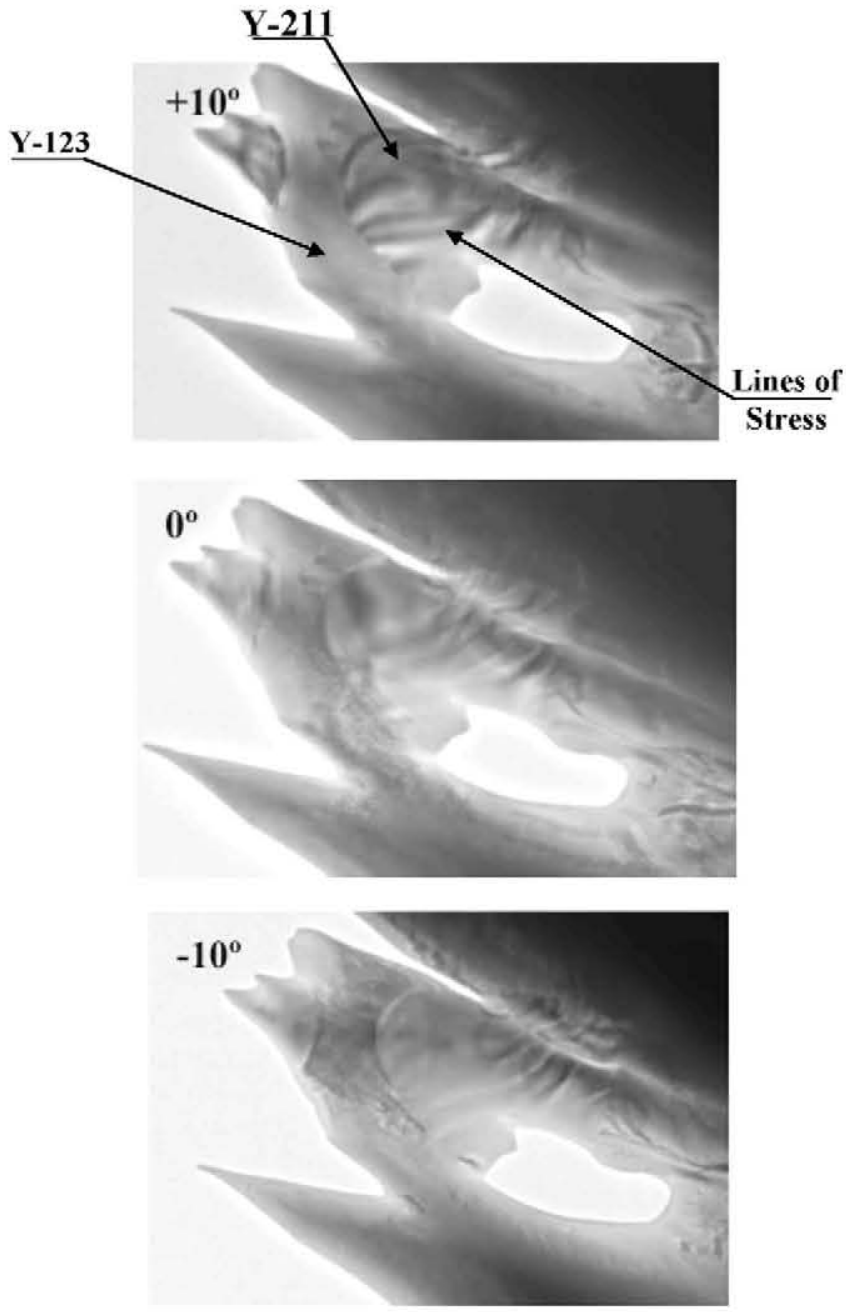

Fig. 8. TEM-images of Y-211 inclusions with different sample orientations (from $10^{\circ}$ to $-10^{\circ}$ ). Residual stresses are evidenced by the elastic deformation of the crystal.

\section{Conclusions}

Nanoindentation and Microindentation techniques were employed in order to determine certain mechanical properties (hardness and elastic modulus) and study the different fracture mechanisms under field stress of $\mathrm{YBaCuO}$ samples textured by the Bridgman technique for use as superconducting materials.

Moreover, the intrinsic hardness of $\mathrm{YBaCuO}$ samples without strain gradient plasticity was determined to be $H_{0}=8.8 \pm 0.3 \mathrm{GPa}$, yielding a constant elastic modulus value of $128 \pm 5 \mathrm{GPa}$. Thus, the Nix and Gao model provides an satisfactory fit for the different experimental points, yielding a linear dependence of hardness with indentation depth in this kind of material.

In addition, observation of the imprints obtained under low loads using nanoindentation and microindentation techniques revealed similar fracture mechanisms, such as chipping and radial cracks at the corners of the imprints.

However, imprints obtained under low loads $(10 \mathrm{mN})$ and observed by FIB exhibited no cracks or failure events under the imprint. 
Lastly, the different TEM images obtained at different angles depicted the evolution of residual stresses inside Y-211 particles. These stresses were responsible for producing several macro- and microcracks around the inclusions.

\section{Acknowledgements}

The corresponding authors would like to thank the Serveis Cientificotècnics (University of Barcelona) for AFM and FIB data, the Linguistic Services at the Universitat de Barcelona for linguistic and stylistic advice, and the Ministerio de Ciencia e Innovación (CSD00C-06-14102 and MAT2009-13979-C03) and the Comunidad de Madrid (S-S2009/MAT-1585-ESTRUMAT2) for their financial support.

\section{References}

[1] D. Koelle, R. Kleiner, F. Ludwig, E. Dantsker, J. Clarke, High-transitiontemperature superconducting quantum interference devices, Rev. Mod. Phys. 71 (1999) 631-686.

[2] L.A. Knauss, A.B. Cawthorne, N. Lettsome, S. Kelly, S. Chatraphorn, E.F. Fleet, F.C. Wellstood, W.E. Vanderlinde, SQUID microscopy for current imaging, Microelectron. Reliab. 41 (2001) 1211-1229.

[3] M.V. Kreutzbruck, in: A.V. Narlikar (Ed.), High Temperature Superconducting 2: Engineering applications, Springer Verlag, Berlin, 2004 p. 223.

[4] J.D. Jorgensen, M.A. Beno, D.G. Hinks, L. Soderholm, K.J. Volin, R.L. Hitterman, J.D. Grace, I.K. Schuller, C.U. Segre, K. Zhang, M.S. Kleefisch, Oxygen ordering and the orthorhombic-to-tetragonal phase transition in $\mathrm{YBa}_{2} \mathrm{Cu}_{3} \mathrm{O}_{7-x}$, Phys. Rev. B 36 (1987) 3608-3616.

[5] R.J. Cava, Oxide superconductors, J. Am. Ceram. Soc. 83 (2000) 5-28.

[6] J.D. Jorgensen, M.A. Beno, D.G. Hinks, L. Soderholm, K.J. Volin, R.L. Hitterman, J.D. Grace, I.K. Schuller, Structural and superconducting properties of orthorombic and tetragonal $\mathrm{YBa}_{2} \mathrm{Cu}_{3} \mathrm{O}_{7-x}$ : the effect of oxygen stoichiometry and ordering on superconductivity, Phys. Rev. B 36 (1987) 5731-5734.

[7] N.I. Tymiak, A. Daugela, T.J. Wyrobek, O.L. Warren, Acoustic emission monitoring of the earliest stages of contact-induced plasticity in sapphire, Acta Mater. 52 (2004) 553-563.

[8] J.J. Roa, X.G. Capdevila, M. Martínez, F. Espiell, M. Segarra, Nanohardness and young's modulus of YBCO samples textured by the Bridgman technique, Nanotechnology 18 (385701) (2007) 1-6.

[9] J.J. Roa, E. Jiménez-Piqué, X.G. Capdevila, M. Segarra, Nanoindentation with spherical tips of single crystals of YBCO textured by the Bridgman technique: determination of indentation stress-strain curves, J. Eur. Ceram. Soc. 30 (2010) 1477-1482.

[10] Y. Yoshino, A. Iwabuchi, K. Noto, N. Sakai, M. Murakami, Vickers hardness of YBCO bulk superconductor at cryogenic temperature, Physica C 357-360 (2001) 796-798.

[11] J. Joo, S.-B. Jung, W. Nah, J.Y. Kim, T.S. Kim, Effects of silver additions on the mechanical properties and resistance to thermal shock of $\mathrm{YBa}_{2} \mathrm{Cu}_{3} \mathrm{O}_{7-x}$ superconductors, Cryogenics 39 (1999) 107-113.

[12] N. Güçlü, U. Kölemen, O. Uzum, S. Selebi, Work of indentation approach for investigation of mechanical properties of YBCO superconductor at cryogenic temperatures, Physica C 433 (2005) 115-122.

[13] H.M. Ledbetter, M.W. Austin, S.A. Kim, M. Lei, Elastic constants and Debye temperature of polycrystalline $\mathrm{Y}_{1} \mathrm{Ba}_{2} \mathrm{Cu}_{3} \mathrm{O}_{7-x}$, J. Mater. Res. 2 (1987) 786-789.

[14] Y.M. Soifer, A. Verdyan, J. Azoulay, M. Kazakevich, E. Rabkin, An AFM study of the morphology and local mechanical properties of superconducting YBCO thin films, Physica C 402 (2004) 80-87.

[15] J.J. Roa, G. Oncins, F.T. Dias, V.N. Vieira, J. Schaf, M. Segarra, AFM as an alternative for Young's modulus determination in ceramic materials in elastic deformation regime, Physica C (2011), DOI: 10.1016/ j.physc.2011.05.249.

[16] K.L. Johnson, J. Mechan, The correlation of indentation experiments, Phys. Solids 18 (1970) 115-126.

[17] J. Alcala, A.C. Barone, M. Anglada, The influence of plastic hardening on surface deformation modes around Vickers and spherical indents, Acta Mater. 48 (2000) 3451-3464.

[18] Z. Burghard, A. Zimmermann, J. Rödel, F. Aldinger, B.R. Lawn, Crack opening profiles of indentation cracks in normal and anomalous glasses, Acta Mater. 52 (2004) 293-297.

[19] J.J. Roa, E. Jiménez-Piqué, X.G. Cadevila, M. Martinez, M. Segarra, Yield strength, shear stress and toughness of YBCO samples textured by Bridgman technique, J. Phys. Conf. Ser. 97 (012116) (2008) 1-6.

[20] B.R. Lawn, M.V. Swain, K. Phillips, On the mode of chipping fracture in brittle solids, J. Mater. Sci. 10 (1975) 1236-1239.

[21] I.G. Serradilla, A. Calleja, X.G. Capdevila, M. Segarra, E. Mendoza, J. Teva, X. Obradors, F. Espiell, Synthesizing the Y-123/Y-211 composite by the PVA method, Supercond. Sci. Technol. 15 (2002) 566-571.

[22] S. Piñol, V. Gomis, V. Martínez, B. Labarta, A. Fontcuberta, X. Obradors, Bridgman growth and enhanced critical currents in textured $\mathrm{YBa}_{2} \mathrm{Cu}_{3} \mathrm{O}_{7}$ $\mathrm{Y}_{2} \mathrm{BaCuO}_{5}$ composites, J. Alloys Compd. 195 (1993) 11-14.

[23] M. Ullrich, H. Walter, A. Leenders, H.C. Freyhardt, Batch production of high-quality-customized-shaped-monolithic HTSC, Physica C 311 (1999) $86-92$.

[24] W.C. Oliver, G. Pharr, Measurement of hardness and elastic modulus by instrumented indentation: advances in understanding and refinements to methodology, J. Mater. Res. 19 (2004) 3-20.

[25] E. Jiménez-Piqué, Y. Gaillard, M. Anglada, Instrumented indentation of layered ceramic materials, Key Eng. Mater. 333 (2007) 107.

[26] I. Horcas, R. Fernández, J.M. Gómez-Rodríguez, J. Colchero, J. GómezHerrero, A.M. Baro, WSXM: a software for scanning probe microscopy and a tool for nanotechnology, Rev. Sci. Instrum. 78 (013705) (2007) 1-8.

[27] S. Sanfilipo, A. Sulpice, O. Laborde, D. Dourgault, Th. Fournier, Experimental evidence of twin-planes pinning in the ab-plane studied in large $c$ axis $\mathrm{YBa}_{2} \mathrm{Cu}_{3} \mathrm{O}_{7}$ samples, Phys. Rev. B 58 (1998) 15189-15197.

[28] S.V. Lubenets, V.D. Natsik, L.S. Fomenko, Plasticity and strength of metal oxide high-temperature superconductors, Low Temp. Phys. 30 (2004) 345-376 (review).

[29] F. Sandiumente, T. Puig, J. Rabier, J. Plain, X. Obradors, Optimization of flux pinning in bulk melt textured 1-2-3 superconductors: bringing dislocations under control, Adv. Mater. 12 (2000) 375-381.

[30] W.W. Gerberich, N.I. Tymiak, J.C. Grunlan, M.F. Horstemeyer, M.I. Baskes, Interpretations of indentation size effects, J. Appl. Mech. 69 (2002) 433-442.

[31] W.D. Nix, H. Gao, Indentation size effects in crystalline materials: a law for strain gradient plasticity, J. Mech. Phys. Solids 46 (1998) 411-425.

[32] Y.Y. Lim, M.M. Chaudhri, Do residual nanoindentations in metals and ceramics relax with time? J. Phys. D: Appl. Phys. 34 (2001) L70-L78.

[33] A. Verdyan, Y.M. Soifer, J. Azoulay, E. Rabkin, M. Kazakevich, Nanohardness and crack resistance of HTS YBCO thin films, IEEE Trans. Appl. Supercond. 15 (2005) 3585-3588.

[34] N.M. Alford, J.D. Birchall, W.J. Clegg, M.A. Harmer, K. Kendall, D.H. Jones, Physical and mechanical properties of $\mathrm{YBa}_{2} \mathrm{Cu}_{3} \mathrm{O}_{7-x}$ superconductors, J. Mater. Sci. 23 (1988) 761-768.

[35] B.R. Lawn, D.B. Marshall, Hardness, toughness, and brittleness: an indentation analysis, J. Am. Ceram. Soc. 62 (1975) 347-350.

[36] Z. Burghard, A. Zimmermann, J. Rodel, F. Aldinger, B.R. Lawn, Crack opening profiles in normal and anomalous glasses, Acta Mater. 52 (2004) 293-297.

[37] P. Diko, N. Pelerin, P. Odier, Microstructure analysis of melt-textured $\mathrm{YBa}_{2} \mathrm{Cu}_{3} \mathrm{O}_{7-x}$ ceramics by polarized light microscopy, Phys. C: Supercond. 247 (1995) 169-182.

[38] D. Hull, D.J. Bacon, Chapter 8: origin and multiplication of dislocations, in: Introduction to Dislocations, fourth ed., Butterworth-Heinemann, (2001), pp. 145-146, ISBN: 0750646810. 